\title{
The Role of Humic Substances in Abiotic Clay Mineral Transformation
}

\author{
Hongyan Zuo and Hailiang Dong
}

Department of Geology \& Environmental Earth Science, Oxford, USA.

Natural Fe-bearing clay minerals occur extensively in soils, sediments and sedimentary rocks. Temperature, pressure, time, and chemical composition of clay minerals are usually considered as variables for clay mineral transformation during pedogenic and diagenetic processes. During the last decade, the role of microbial activities in promoting clay mineral transformations has been recognized and proved thoroughly. However, other pathways may be possible to promote such clay reactions. For example, humic substances and clay minerals are frequently found to co-exist in anoxic, water-saturated environments. Little is known about the role of humic acid in clay mineral transformation and secondary mineralization, even when microbial activity is absent.

To test the hypothesis that humic substances can promote clay mineral transformations, we conducted a laboratory experiment with well-characterized clays and humic acids. Nontronite (NAu-2, a size fraction of 0.05-0.2 $\mu \mathrm{m}$ ) and Leonardite humic acid (LHA) or Pahokee Peat humic acid (PPHA) were prepared in a buffered system (30 mM bicarbonate buffer, $\mathrm{pH} 7)$. Anoxic NAu-2 and humic acids were sterilized by autoclaving and filtering $(0.22 \mu \mathrm{m})$, respectively. NAu-2 was mixed with either LHA or PPHA to achieve a final concentration of $3.75 \mathrm{~g} / \mathrm{L}$ and $2.5 \mathrm{~g} / \mathrm{L}$, respectively. Mixtures were incubated for a month on a shaker. Mineralogical changes were characterized with X-ray diffraction (XRD) and scanning electron microscopy (SEM).

XRD results on incubated samples detected secondary minerals including talc and illite, possible albite with LHA treatment. Sample collected from NAu-2-LHA (2.5 g/l) mixture was chosen as a representative to further study abiotic mineral transformations induced by humic substances. The presence of humic substances promoted NAu-2 particle aggregation, consistent with a previous study (Liu et al., 2016) (particle 1 in Fig.1). The corresponding EDS spectrum showed a typical composition of Na-saturated NAu-2 ( $\mathrm{Si}, \mathrm{O}, \mathrm{Fe}, \mathrm{Al}$, and $\mathrm{Na}$ ), indicating no major compositional change in the aggregation process. Other particles on the same image showed a decreased amount of Fe and uptake of $\mathrm{K}$ (Particle 2 in Fig. 1). K uptake may be a charge-compensating mechanism for Fe dissolution. In some particles (Particle 3), a crinkled morphology and a complete loss of Fe peak was observed. Particle 4 showed a similar composition to Particle 3, however, the morphology and the composition are indicative of euhedral albite.

Another mineral transformation pathway is from nontronite to talc. The Al peak in particle 2 decreased (Fig.2A) relative to particle 1 (aggregated $\mathrm{NAu}-2$ ), and correspondingly, $\mathrm{Mg}$ was taken up to balance the charge. Finally, Fe-bearing talc formed without structural Al (particle 3 in Fig.2A). The neoformed talc displayed an euhedral platy morphology with sharp edges. Some large talc crystal $(>6 \mu \mathrm{m})$ were observed (particle 5 and 6 in Fig.2B). An elongated silica particle was observed in association with talc (Particle 4 in Fig.2B).

References:

[1] Liu D et al, Chemical Geology 447 (2016), p. 199. 

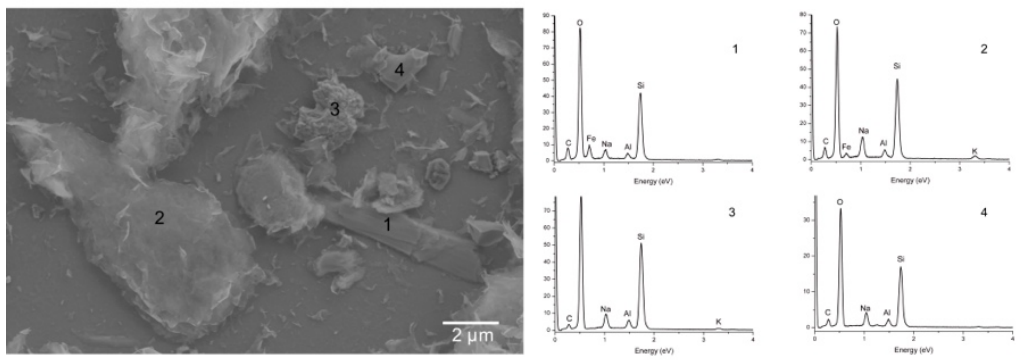

Figure 1. SEM images of NAu-2 after reaction with humic substances. Particle 1 shows a larger size (6 $\mu \mathrm{m})$ than original NAu-2 size $(0.05-0.2 \mu \mathrm{m})$, indicating aggregation induced by humic substances. The EDS spectrum of Particle 1 shows $\mathrm{Fe}, \mathrm{Al}, \mathrm{Na}, \mathrm{Si}$, and $\mathrm{O}$ peaks, a typical composition of NAu- 2 . The EDS of Particle 2 shows a K peak but with a decreased amount of Fe relative to Particle 1. A crinkled morphology is shown in Particle 3 structural Fe loss is totally lost. A neoformed albite with an euhedral shape is displayed in Particle 4.
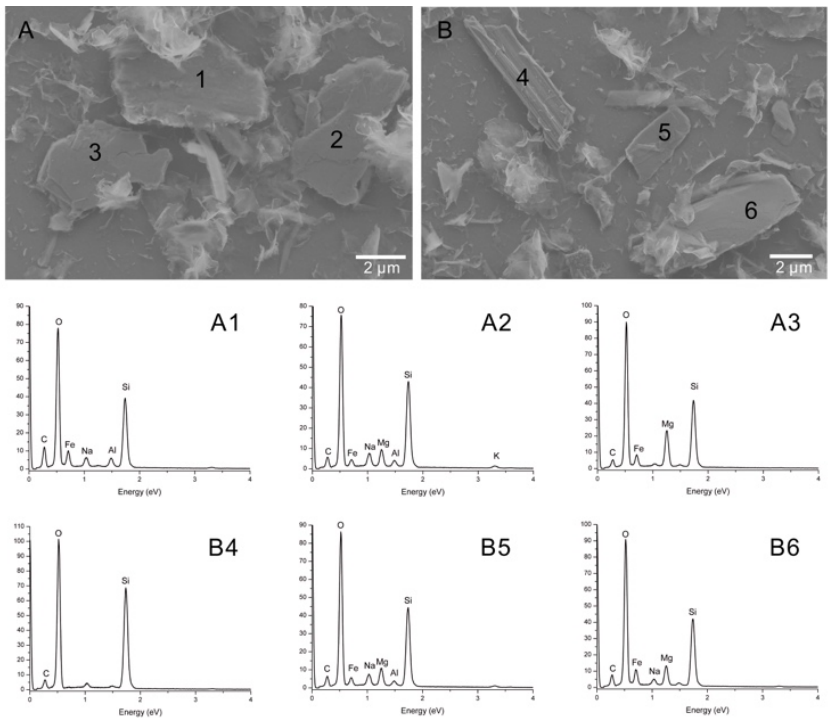

Figure 2. SEM images of NAu-2 and its transformation to talc: (A) Particles 1-3 showing the change from NAu-2 to talc where particle 2 shows an intermediate composition with a decreased Al and increased Mg; (B) Particle 4 showing an elongated silica particle, Particles 5 and 6 display crystals of 4-6 $\mu \mathrm{m}$ in size. 